\title{
Randomized comparative study between classic laryngeal mask airway and I gel Airway in obese patients having BMI 35-40 during elective non- abdominal surgery
}

\author{
Tamer Mohamed Khair \\ Cairo University \\ Mohamed Ibrahim Belita \\ Cairo University \\ Ahmed Abdelaziz Seleem \\ Cairo University
}

Ehab Mohi Atta

Cairo University

Ahmed Essam Salem

Tanta University Faculty of Medicine

Safinaz Hassan Osman

Cairo University

Norhan Abdelaleem Ali

Cairo University

Ahmed Farag

Cairo University

Ahmed Shaker Ibrahim

Cairo University

Reham Ali Abdelrahman

Cairo University

Rania Samir Fahmy

Cairo University

Shady Rady Abdalla

Cairo University

Nadia Yossif Helmy

Cairo University

Fatma Ibrahim Elsayed

Cairo University

Ahmed Abdalla Mohamed ( $\sim$ ahmed.aboali7268@gmail.com )

Cairo University https://orcid.org/0000-0002-3024-7974 


\section{Research article}

Keywords: Classic laryngeal mask airway, I gel, Supraglottic devices, obesity, Time of insertion, leaking pressure

Posted Date: April 12th, 2019

DOI: https://doi.org/10.21203/rs.2.9123/v1

License: (c) (1) This work is licensed under a Creative Commons Attribution 4.0 International License. Read Full License 


\section{Abstract}

The authors have removed this preprint from Research Square. 